\title{
Research
}

\section{Practice nurses and integrated care examined}

Sheila Stark, Tony Warne and Clare Street The Manchester Metropolitan University, Faculty of Community Studies, Law and Education, Department of Health Care Studies, Manchester, UK

\begin{abstract}
Primary Care Groups and Primary Care Trusts have the task of achieving an integrated health care service, serving local communities. A regional Education and Training Consortium commissioned a 1-year study to provide information in order to develop a workforce strategy that aimed to achieve this agenda. The study involved individuals who plan, provide and/or manage primary and community health care service in three Health Authorities, working in partnership. The three phases of data collection included focus groups, individual interviews and a survey, which produced qualitative and quantitative data. The findings outlined in this paper primarily focus on practice nurses and integrated care. Several tensions emerged in this context which could inhibit the development of integrated care. These included conflict within professional relationships, inequality in pay and working conditions, a lack of understanding of how integrated care would or could translate into practice and turbulent environmental contexts. We argue, these tensions often created gaps between policy, practice and professional discourses which, unless acknowledged and understood may result in integrated care remain a health, but unachievable, rhetoric.
\end{abstract}

Key words: integrated care; practice nurses; local communities

\section{Introduction}

The White Paper The New NHS: Modern, Dependable (DoH, 1997) set out the agenda for modernizing the National Health Service (NHS) by working towards integrated health care services, serving local communities. Subsequently, these proposals have been underpinned by further policy documents outlining the structural and functional changes necessary to achieve this goal. These include Saving Lives: Our Healthier Nation (DoH, 1999a), A First Class Service (DoH, 1998a), Information for Health Care (NHSE, 1998a), Working Together - Securing a Quality Workforce for the NHS (NHSE, 1998b), Making a Difference (DoH, 1999b) and more recently The NHS Plan (DoH, 2000). Amongst other issues,

Address for correspondence: Dr S. Stark, The Manchester Metropolitan University, Faculty of Community Studies, Law and Education, Department of Health Care Studies, Elizabeth Gaskell Campus, Hathersage Road, Manchester M13 0JA, UK. these policy documents identify the need to explore the role of staff working in primary health care and the contribution they can make to realizing the new agenda. The study discussed in this paper was commissioned by a regional Education and Training Consortium and included three Health Authorities working together in partnership, with the intention of addressing issues arising out of this NHS agenda. The commissioners were interested in exploring the role of nurses in primary health care, in particular practice nurses (PNs), in order to provide more integrated care. Data were collected and analysed over 1 year. Issues of role definition, recruitment and retention, teamworking, and the continuing professional development of nurses in primary health care were examined. There appears, however, to be a number of potential gaps between policy, practice and professional discourses in primary health care which may inhibit the current agenda being realized. These will be discussed in this paper. 


\section{Setting the context}

Implementation of governmental policy relies on effective human resource management (HRM) within the operating environment (Iphofen and Poland, 1998). However, despite the NHS being one of the largest employers in the United Kingdom, HRM has only recently attracted the strategic and operational overview it has perhaps demanded (Rivett, 1998). This has been particularly true with respect to the largest workforce group, the nurses (Noakes and Johnson, 1999). Although the United Kingdom may not be undertaking the large-scale regional collaborations for nursing workforce development that are currently being undertaken in the United States (Rapson and Rice, 1999) valuable work is apparent. For example progress has been made in the areas of recruitment and retention (Atkin and Lunt, 1996; Smadu, 1996), developing a more flexible, integrated and multi-skilled workforce (Bull, 1998; Gerrish, 1999; Pong, 1997) and expanding the scope of training and development (Choppin, 1997; Hyde and Wright, 1997; Steel, 1999).

Furthermore, in primary health care, it appears that governmental intentions have been heard. Research into the development of this area reinforces a multi-professional, multi-agency, integrated approach to service provision (Black and Hagel, 1996; Kernick, 1999; Pearson and Spencer, 1997; Ross and Mackenzie, 1996; Wilcox and Ruffell, 1997; Warne, 1998; Young, 1997). However, as Kernick (1999) notes, 'when external factors dictate change, adjustment within a profession occurs but remains within an established framework' (p. 648). This begs the question: will the formation of Primary Care Groups (PCGs) and Primary Care Trusts (PCTs) provide the opportunity (demand?) for workforce planning strategies to be developed within new frameworks, which promote integrated care? Miller etal. (1999) believe progress towards a primary care-led NHS will be 'patchy' due to the GPs attitudes to resources and the new role being carved out for them. Lewis (1999) shares similar concerns over the dominant position of GPs persisting within the PCG/PCT structure.

The study outlined in this paper was commissioned in July 1998, prior to the formation of PCGs and concluded in July 1999, by which time PCGs were a reality. The findings provide insights for PCGs/PCTs, Health Authorities and individual health care professionals, with respect to experience, expectations and emerging organizational conditions vis-à-vis teamworking and integrated care for PNs and other health care professionals.

\section{The study}

The aims of the study were, first, to develop a clearer contextual understanding of nursing primarily in general practice, but also primary health care in general, in three health authorities, centring specifically around workforce planning issues of role boundaries, professional development, attitudes towards and experiences of integrated teamworking, contextual changes and so on. Second, but linked with the first aim, was to evaluate a training practice initiative for general PNs in one of the Health Authorities. To what extent was this initiative an effective professional development route for introducing nurses into primary care and addressing recruitment and retention? (The findings of this evaluation have been written elsewhere (Stark et al., 2000) and will not be discussed in this paper.) Finally, to use these insights and understanding in recommending a way forward for the development of a workforce plan for the role of nurses in general practice. However, since the role of the nurse in general practice cannot and should not be discussed in isolation from other professionals involved in primary health care, particularly in light of integrated care, the role/s of other nurses in this area are also included in subsequent discussions.

\section{Methodology}

The study consisted of three main aspects, which formed an incremental and interconnected cycle of both data collection and analysis.

\section{Aspect 1, literature review}

Literature, including policy documents, enabled us to keep informed of the rapid contextual changes taking place nationally. The study took place in what organizational theorists call a 'turbulent' environment (Emery and Trist, 1965), 
Table 1 Primary care focus groups

Focus group 1, 9

Focus group 2

Focus group 3, 10

Focus group 4, 11

Focus group 5, 12

Focus group 6

Focus group 7

Focus group 8
8 members (PNs, DNs, HVs, Primary Care Development Manager)

7 GPs

10 members (PNs, Nurse Clinician, DNs, Trainee PN, Practice Manager, HV)

9 members (PNs, DNs, HVs, Practice Managers, Specialist Nurse)

14 qualified nurses undertaking a Community Health Degree

5 members (PCG Nurse Board Member, PNs, DNs)

5 members (PNs, Practice Manager, DNs)

8 members (PCG Nurse Board Members) such a context provided the opportunity to collect data at a highly significant time in the NHS.

\section{Aspect 2, focus groups and interviews}

Twelve focus groups, averaging eight to nine participants, together with 16 individual interviews were undertaken over a period of 8 months (see Tables 1 and 2). Each focus group and interview lasted 40-60 min and were tape recorded following consent by the participants. In addition to these data collection sessions we gained several useful insights from numerous informal conversations with individuals both face to face and via the telephone. Fieldnotes were written after informal observational data were collected as a result of several visits to clinical areas.

Some focus groups and interviews predominantly focused on the evaluation of the training practice initiative, while others addressed broader workforce issues with staff working in various clinical settings. Experiences and attitudes towards teamworking and leadership, perceptions of roles, training and professional development, organizational culture and change including the introduction of PCGs, are examples of some of the issues discussed within a semi-structured format. The findings facilitated the development of the survey used in aspect 3 .

\section{Aspect 3, survey}

Two hundred and ten surveys (70 in each Health Authority) were distributed to a cross-section of staff working in the planning, provision and management of primary health care services in each of the three Health Authorities. An average response rate of $46 \%(n=96)$ was achieved (since mail surveys commonly produce a low response (Herberlein and Baumgartner, 1978; Kanuk and Berensen, 1975) the team were satisfied with this response rate; a response of less than $20 \%$ is commonly felt not to be representative). The survey provided an effective means of informing health care professionals about the attitudes and behaviours of others

Table 2 Primary care individual interviews

Interview 1, 2, 6, $10,14,16$

Interview 3

Interview 4, 13

Interview 5

Interview 7

Interview 8, 15

Interview 9

Interview 11

Interview 12
PCG Nurse Board Members

Director of Planning

Primary Care Development and Training Manager

Director of Nursing (Community)

Nurse Manager Community Services

PN Development and Support Manager

Primary Care Manager

DN Team Leader

Nurse Prescribing Course Tutor 
working in primary care and, further, encouraged participants to comment upon them. The survey provided mainly quantitative data, but additional comments also provided qualitative data. The results of the survey provided a focus for a final series of focus group and interviews. Participants in the first round of focus groups and interviews were revisited where possible. These sessions were used to 'test' the validity of the teams' interpretations of the data collected.

\section{Sample}

The target group comprised of individuals involved in planning, providing and/or managing primary health care services in the three Health Authorities. Although this was predominately nurses, representatives from a range other professional groups formed part of the overall group of respondents (see Tables 1 and 2). Several individuals at management levels were involved in individual interviews, while practitioners often preferred to meet in focus group settings. Over $50 \%$ of the survey respondents were PNs, 24\% GPs and the remaining $26 \%$ comprised of other health professionals such as health visitors (HVs), district nurses (DNs) managers, PCG board members, specialist community nurses, etc.

\section{Analysis}

There was a high degree of congruence across the responses from each Health Authority, as well as from the methods used to collect the data. Thus, it was decided that the three Health Authorities could be treated as one sample in the analysis. The qualitative data from the focus groups, individual interviews, field notes and the descriptive data from the survey were analysed during the fieldwork phase and again when all the data had been collected. The transcripts and texts were read and reread and emerging themes were used to guide the data collected during the subsequent aspect/s.

The quantitative responses from the survey were calibrated using a predetermined ordinal scale based upon bipolar agreement factors (ranging from 'strongly agree' to 'strongly disagree'). The subsequent data were then tallied and collated. Descriptive statistics were used, in particular percentages were calculated for each response. Separate percentages were calculated for the professional status variable, since it became apparent this produced some interesting results.

\section{Findings}

This paper focuses on the findings in relation to PNs and integrated care. A number of themes were identified.

\section{Roles and responsibilities}

Respondents were asked with whom they directly worked as part of their team. Table 3 represents the response (note our sample outlined above, i.e., over $50 \%$ of the respondents were $\mathrm{PNs})$. It indicates, therefore, health professionals who will have a responsibility for integrated care. Of the respondents, $95 \%$ felt they understood their role within their team, while $64 \%$ of the respondents felt that others understood their role. When GP returns were removed, only $41 \%$ of the nurses felt others understood their role (see Gerrish, 1999, for a further discussion of poor knowledge of other professional roles). Reasons for a misconception of roles generally fitted into one of three categories:

\section{1) A blurring of roles - especially between PNs and DNs}

I don't think that some of the GPs know the difference between a D grade role and an E grade role ... they actually said to me, 'why can't she go out and do that' ... there was

Table 3 Individuals with whom respondents directly worked

\begin{tabular}{lll}
\hline DN & $91 \%$ & $(87)$ \\
GP & $79 \%$ & $(75)$ \\
HV & $78 \%$ & $(74)$ \\
PN & $75 \%$ & $(72)$ \\
Practice Manager & $75 \%$ & $(72)$ \\
Midwife & $60 \%$ & $(57)$ \\
Health Care Assistant & $20 \%$ & $(19)$ \\
Community Psychiatric Nurse & $18 \%$ & $(17)$ \\
Nurse Practitioner & $16 \%$ & $(15)$ \\
\hline
\end{tabular}

One or two respondents mentioned 15 other team members

Number of respondents in brackets 
no understanding of face level issues and responsibilities.

$(\mathrm{PN})$

The GPs just think you're a nurse.

$(\mathrm{PN})$

Some overlapping of roles with DNs causes some confusion for other disciplines.

$(\mathrm{PN})$

Whilst a blurring between nursing roles was stated, perhaps there was also an unacknowledged state of flux within the PN role.

This is a relatively newly created role and as yet is evolving, therefore, it is difficult to assess understanding of it at this point.

$(\mathrm{PN})$

\section{2) A general lack of communication}

Sometimes I think we work in our own little area and we're not aware of skills other people have. We take it for granted that because you are a DN you have such-andsuch skill, but I guess we don't know. We don't talk about it.

(DN)

\section{3) Idealization of the role}

For example, nurses talked about PNs having 'autonomy' and 'flexibility', while several PNs themselves talked of 'isolation' and 'domination' by GPs.

While there was evidence that individuals clearly drew and defended professional boundaries vis-à-vis roles and responsibilities (similar finding to Northway and Walker, 1999), 63\% of the participants saw the potential for primary health care nurses to share more work:

To have individual people with their experiences, but to have a blurring of the boundaries for certain things, this is a much more sensible approach.

(PN)

What these boundaries were, however, was often difficult to establish with the participants. Furthermore, which particular boundary/ies they felt they could/would cohabit with others was also unclear.

\section{Integrated care $=$ role sharing $=$ generic worker}

Often, providing an integrated approach to care was translated by the nursing participants into role sharing and the development of the generic worker.
This begs the question as to the participants' understandings and interpretations of the terms 'integrated teams' and the 'generic worker'.

Integrated teamwork is desirable, but not at the expense of knowledge or expertise within existing roles. It should not be a cheap option.

(HV)

Each has its own specialism/strengths. It would be difficult to have a large knowledge base about many things - this would need to happen if roles were shared.

$(\mathrm{PN})$

This notion of a generic health care worker replacing DNs and PNs is a load of rubbish ... I've seen it [sharing work] in practice and it caused such a hassle between the District Nursing team and the practice as a whole.

(Practice manager)

While the concept of integrated care was talked about in positive tones, the translation of the concept into practical realities, for their role in particular, was articulated in more negative tones often in terms of things being 'added on' to their current practice. However, the expansion of a primary care health care assistant role (potentially taking away from their 'load' as opposed to adding to?) was seen as a possibility.

That's right, I think a lot of PNs do jobs that doesn't need a PN to do it.

\section{(PCG Nurse Board Member)}

So, a conflicting discourse emerged (a gap between theory and practice?) when nurses discussed integrated care. It appeared there was a taken-for-granted assumption everyone 'knew' what integrated care was and how it could be translated into practice. However, when probed, our findings revealed an unclear (and unspoken, perhaps because unrecognized) conception of what integrated care would, could or should look like. This resulted in nurses retreating into the safety of their professional specialisms, talking about their importance as a uniprofessional and, as we saw earlier, criticizing the role of a generic worker.

Being a HV means that I work with children. I pass them on when they get older, and there is good communication across the team. But I can change a dressing just as well as any- 
body else. Nobody wants me to do that, so the result is that a couple of us can be going to the same house at the same time.

(PCG Nurse Board Member)

On occasions there have been problems with DNs. They refuse home visits to do blood tests/BP checks, etc., and refer work to me but then get upset if I'm seen to be taking work away from them.

$(\mathrm{PN})$

Conflict with other health care professionals over role boundary demarcation and inequalities over terms and conditions of pay have been discussed elsewhere in the literature (Hirst et al., 1995; Gerrish, 1999) and surfaced with the participants in this study. This arose, in particular when they were asked to consider obstacles to effective teamworking.

\section{Obstacles to effective teamworking in primary care}

Survey participants were asked to respond to statements made in the focus groups and interviews in relation to obstacles to primary care teams working effectively. Table 4 highlights the aggregated responses from the nurses and the GPs. Table 5, however, shows a different response when the data were analysed separately for these two groups.

The nurses agreed that all the issues were obstacles to effective teamworking - even the low-

Table 4 Obstacles to primary care teams working effectively

\begin{tabular}{llll}
\hline & $\begin{array}{l}\text { Strongly } \\
\text { agree } \\
\%\end{array}$ & $\begin{array}{l}\text { Neutral } \\
\%\end{array}$ & $\begin{array}{l}\text { Strongly } \\
\text { disagree } \\
\%\end{array}$ \\
\hline $\begin{array}{l}\text { Unco-ordinated } \\
\text { information } \\
\text { systems }\end{array}$ & $74(71)$ & $14(13)$ & $12(11)$ \\
$\begin{array}{l}\text { Ineffective management } \\
\text { Varying conditions of }\end{array}$ & $66(63)$ & $21(20)$ & $13(12)$ \\
$\begin{array}{l}\text { service } \\
\begin{array}{l}\text { Different professional } \\
\text { foci }\end{array}\end{array}$ & $53(50)$ & $28(26)$ & $11(10)$ \\
$\begin{array}{l}\text { Lack of a shared } \\
\text { workplace }\end{array}$ & $53(50)$ & $24(23)$ & $23(22)$ \\
$\begin{array}{l}\text { GP domination } \\
\text { Unequal pay scales }\end{array}$ & $51(48)$ & $30(28)$ & $19(18)$ \\
$\begin{array}{l}\text { Different knowledge } \\
\text { bases }\end{array}$ & $30(38)$ & $31(29)$ & $29(27)$ \\
\hline
\end{tabular}

The number of respondents is in brackets
Table 5 Primary care nurses and GPs' views to the obstacles to primary health care teams ranked in order to agreement (highest first)

\begin{tabular}{|c|c|}
\hline GPs & Nurses \\
\hline Ineffective management & $\begin{array}{l}\text { Unco-ordinated } \\
\text { information systems }\end{array}$ \\
\hline $\begin{array}{l}\text { Unco-ordinated } \\
\text { information systems }\end{array}$ & Ineffective management \\
\hline Lack of a shared workplace & $\begin{array}{l}\text { Varying conditions of } \\
\text { service }\end{array}$ \\
\hline $\begin{array}{l}\text { Varying conditions of } \\
\text { service }\end{array}$ & GP domination \\
\hline $\begin{array}{l}\text { Different professional foci } \\
\text { Different knowledge bases }\end{array}$ & $\begin{array}{l}\text { Different professional foci } \\
\text { Lack of a shared workplace }\end{array}$ \\
\hline Unequal pay scales & Unequal pay scales \\
\hline GP domination & Different knowledge bases \\
\hline
\end{tabular}

est ranked obstacle (different knowledge bases) had $41 \%$ of the nurses believing it was an obstacle. The majority of the GPs, however, agreed only half were obstacles. More than 50\% actively disagreed that GP domination, unequal pay scales and different knowledge bases were obstacles to effective teamworking. Given that these professionals are 'team members', these conflicting views could hinder integrative teamworking. Ineffective management and unco-ordinated information systems were perceived to be the main obstacles for both groups.

We do not need petty management - it gets in the way of work and job satisfaction. (PN)

While our data revealed that the majority of personal experiences with effective teamworking were negative, there were exceptions.

In this practice there is a good understanding of all our roles, but we had to work at it. (PCG Nurse Board Member)

Our GPs are very supportive of our role development, and although they might ask us to do something, if we feel we don't have the right skills for that we can discuss it and its not an issue ... the GPs and nurses all work as a team, sharing experience . . . but we have had a very stable team now for many years so maybe that's why.

\section{(PCG Nurse Board Member)}

Stability, rapport and good communication between team members seemed to enhance team- 
working. This is echoed in current literature on teamworking in nursing (Norman et al., 1998; Miller et al., 1999; Stark et al., 2000). It could be claimed this is simple rhetoric, but apparently extraordinarily difficult to achieve in practice. We took a closer look at some of the perceived obstacles to effective teamworking.

\section{A lack of parity between nurses}

We need to sort out terms and conditions of employment for PNs. There are too many different rates of pay at the moment. This might be more possible with PCGs.

(Primary Care Development and Training Manager)

I would like to see DNs, HVs and PNs working together as a unit, sharing skills and holding their own budgets.

(GP)

Many would argue that for nurses to work effectively together as a unit, as described by the GP above, there should be parity in the qualification status of HVs, DNs and PNs.

All [PNs] should be required to undertake an accredited PN qualification to raise standards and bring about equality between HVs, DNs and PNs.

(GP)

Currently, there is often a lack of parity in pay and conditions between PNs and between PNs and other community nurses, such as DNs and HVs. If PNs were employed under common terms and conditions of employment would this facilitate more effective skill mix assessments and increase the possibility for greater integration and role sharing? On the other hand, in a free labour market, pay differentials often reflect differences in the nature of the job, the availability of potential employees and even issues such as the attractiveness of the work environment. Linked with discussions of parity of pay and conditions is often the chant for a single employer for all nurses working in primary and community care. Irrespective of the current infrastructures not being in place to support such a move, would a single employer resolve issues of ineffective teamworking and issues of parity of pay and conditions? It is perhaps worth noting that while the Royal College of Nurs- ing (1999) estimates that there is a shortage of nurses in the region of 13000 and an average vacancy rate of $12 \%$ (Roberts and Fielding, 1999) the numbers of nurses entering practice nursing has trebled over the last 10 years (Kernick, 1999). This begs the question: do the benefits of practice nursing, such as job flexibility, sociable work hours and a certain amount of autonomy in practice, outweigh problems of lack of parity with other nurses?

A persistent reason for PNs wanting parity is due to the GP domination many experience. This had been well documented in the literature (Field and West, 1995; Gerrish, 1999; Oughbridge, 1998; Thomas and Corney, 1993) and was reinforced by respondents in the survey $(59 \%$ of the nurses felt GP domination was a barrier to effective teamworking). Our discussions with participants, however, revealed more positive relationships with their employers (GPs). Thus, highlighting a potential gap between the theory (i.e., GP domination exists) and the actual domination experienced.

\section{Nurses' perceptions of GP domination}

Dialogue with nurses in the focus groups and interviews revealed that nurses believed, but did not necessarily experience, GPs to:

1) be the dominant force in developing and maintaining the culture of the practice team;

2) direct work of individuals (either limiting their role or expanding it beyond what the PNs felt they were legally safe to practice);

3) influence (again, often limiting) the training and development of PNs.

The lack of equity between GPs in relation to terms and conditions of employment of the PNs, further caused unrest amongst the nurses: $64 \%$ of nurses and, perhaps more surprisingly, $50 \%$ of GPs felt GP domination was possibly the main factor PNs left their positions.

As a PN you do what your GP says because your GP is your employer.

$(\mathrm{PN})$

Some practices treat their PNs appallingly and they work in very poor conditions. But word gets out and eventually no one will work for them.

(Primary Care Development and Training Manager)

Primary Health Care Research and Development 2002; 3: 11-21 
Since GPs are the employers and PNs the employees, would it not be reasonable that as an employer they have a right to say what they want their employees to do with their time and to control how much time they are away from the practice undergoing professional development? Perhaps a closer examination of how the term 'domination' is being used by nurses may prove insightful. Given the examples of potential domination outlined above, does the same 'domination' not exist with other employers, such as Community Trusts? If GP domination is a problem, why are so many nurses entering general practice? Furthermore, there is no evidence that practice nursing has a high attrition rate. In this study, 95\% of the GPs felt their nurse was a valuable member of the primary care team compared with $56 \%$ of the nurses. This response reinforces the opinion that nurses are not proactive in promoting their professional status (Walsh, 1998), or that GPs have not effectively communicated their employees worth to them. The nurses in this study possibly reinforced the latter since many commented that they did not receive annual performance reviews.

The majority of PNs in this study had been in their post for several years and saw their team, metaphorically, as an 'arranged marriage' (49\%), or as a 'family' (33\%, interestingly $70 \%$ of GPs described their team as a family), but not a warring tribe $(0 \%)$. Further, several positive benefits of working with their GP emerged.

\section{1) Opportunity to shape their role according to their interests}

I sometimes don't know if the younger ones [GPs] realise what we are capable of, but the older ones do ... I think they have realised that we are a big group really and have taken quite a lot of things off them.

(PCG Nurse Board Member)

As a PN I enjoy being independent, and I like the fact that I haven't got managers breathing down my neck, telling me to do this and that and the other.

$(\mathrm{PN})$

\section{2) Flexible working hours}

I've just come in this morning, my friend's died and the funeral is on Monday. You just take the time off and come back in ... as long as they see you're working when you're here they're very supportive in that respect.

$(\mathrm{PN})$

\section{3) Support and loyalty}

I think perhaps, working in general practice you become very loyal and perhaps looking at sickness issues a lot more. We know that if we go off sick the reception staff and the practice managers have got like full lists to get rid of ... so you want to drag yourself into work if at all possible.

$(\mathrm{PN})$

I consider career development opportunities are infinite and related to specialist interest, i.e., asthma, diabetes, family planning, etc.

(PN)

Our data, therefore, revealed some incongruency between GPs' domination which the nurses talked about in general terms and their actual experiences. A further theory practice (or expectation-experience) gap?

\section{Management issues}

Ineffective 'management' from either the Health Authority or Community Trusts was a consistently reported factor by the respondents, who felt it was instrumental in the often poor working relationships of the primary care team. Twenty-three per cent of the GPs strongly believed it would be advantageous for all community nurses to be employed by PCGs, while 36\% strongly disagreed with this, but overall, the respondents were relatively evenly represented in their views $(42 \%$ were in favour, $30 \%$ against and $28 \%$ neutral).

One employer for all, greater sharing of skills, self-managed nursing teams, innovative projects, challenging traditional way of working.

(Primary Care Development Manager)

Indeed, most nurses in the survey hoped that being managed by the same body would create more equality between pay and conditions, training and development.

I would like to see an end to direct employment of PNs by GPs. I feel this relationship has a stultifying affect on their ability to see 
the underlying Public Health agenda and where they fit into the picture.

\section{(HV and PCG Board Member)}

Returning to an earlier point, if nurses were to have the same large employer, such as Community Trusts, would they be afforded the same opportunities of flexibility and innovation which smaller organizations, such as general practices, can often provide, or would they have to streamline their workforce according to, and because of, the grade structure? Would nurses be willing to trade this freedom for parity in pay?

With the advent of PCGs and nurse board members, several nurses saw an opportunity to share a leadership role alongside GPs. Others were more sceptical and felt that GP domination would still prevail within PCGs. GPs were unsure whether PCGs would be able to identify PN training needs, on balance they felt they would not.

I think it is a pity that nurses from district nursing, health visiting and practice nursing are not specified. I know there are other nurses, but these are the largest group in community care.

(GP)

Some respondents felt that while a more structured approach to multi-professional teamworking, as envisaged by the creation of PCGs, was a good thing, they had concerns that the different forms of skill mix would result in a dilution of specialisms. The latter echoes an earlier theme of viewing teamworking in generic role terms.

Other management issues centred on issues of uncoordinated information systems, professional isolation of primary care nurses and dispersed 'home bases', which hinder effective integrated teamworking. Both of these issues could be tackled using computer technologies. 'Virtual' teams are still in their infancy within the NHS, but the recent information strategy for health services, Information for Health (DoH, 1998b) suggests there is great potential for this technology to integrate care more effectively. Team members can assess the same data bases and 'talk' to each other regularly. Alpay et al. (2000), however, suggests nurses in particular, may experience challenges as they seek to close the gap between the reality of today and the ultimate vision for information technology in primary health care.

\section{Discussion}

The findings highlight difficulties of working conditions and professional relationships, in achieving more integrated care in primary health care. Many of the findings resonate with other studies carried out over recent years in similar areas (Atkin and Lunt, 1996; Atkin et al., 1994; Bradshaw, 1999; Bull, 1998; Cain et al., 1995; Gerrish, 1999; Kernick, 1999; Rapson and Rice, 1999; Rashid et al., 1996; Steel, 1999; Thomas, 1997; Wiles and Robinson, 1994; Young, 1997).

Traditional and historical professional role differentials are a common feature amongst primary health care teams. A culture of 'competition' versus 'co-operation' still exists and a 'blame' culture is evident between (1) nurses and (2) GPs and nurses, whereby each blames the other for the more negative aspects of the current situation. Inequality between team members appears to be an issue for many PNs who see themselves lower down the 'pecking order' in terms of job status and pay. The reality, however, is that team members are not equal and, many would argue, nor should they be. While there are inequalities in pay between PNs and other community nurses, it could be argued their rewards come in other ways such as job flexibility, autonomy, social working hours, a 'family' type work environment and so on. Furthermore, would equality encourage more effective teamworking? Recently the Oxford Heart Centre at the John Radcliffe Hospital has been subject to an independent enquiry, which warned the NHS that a clash of big egos between consultants was seriously affecting the centres international reputation (Laurence, 2000). This suggests that effective teamworking needs more than the issues of equality to be addressed.

There appears to be a lack of understanding relating to what integrated care could or should look like in practical terms. Further, there is a lack of understanding of the PNs role and that of other primary care professionals. From our data, these factors appeared to result in a series of potential gaps between theories and practices, expectations and experiences, which may militate against integrated care. We suggest health professionals need a better understanding of the realities of practice, learning how to read the distribution of power and authority in groups and how to manage it. Further, we raise a cautionary note if policy-makers are 
looking for a prescription of 'best practice' in relation to integrated care. This could lead to overgeneralizations in contexts where this 'best practice' may not fit. Primary health care is diverse and shifting; we believe such diversity should be embraced if health care is to meet local needs. Furthermore, unless the politics of the context are recognized (professional status, competition, hierarchies, uncertainty of roles, etc.), then integrated care may remain a utopian smoothing mechanism for the turbulence in primary health care. Such utopian vision can leave professionals cynical as they fail to reach policy ideals.

Finally, could the development of 'virtual' teams lead to improving integrated care? Perhaps Health Authorities need to look for information technology specialists as key team players. They may create significant opportunities that empower team members to work together more effectively than would purpose-built buildings or charismatic health care team managers.

\section{Summary}

PCGs/PCTs have the task of achieving the national primary care policy objectives within a turbulent context as described at the beginning of the paper. This requires HRM strategic planning, based on information that ensures professional and organizational, as well as local health needs are met. This study begins to provide information to local stakeholders vis-à-vis workforce issues related to achieve more integrated care. We suggest that negative aspects of the existing organizational culture (power relations, uncertainties, stereotypes and professional rivalry) may militate against NHS policy objectives being achieved. We suggest the realities of the context need to be acknowledged and understood in order to enable terms such as integrative care and teamworking, to be more than a healthy, but unachievable, rhetoric.

\section{Acknowledgements}

The research team thank the Merseyside Education and Training Consortium for commissioning this study. In addition, thanks are extended to the participants from St Helens and Knowsley, Sefton and
Liverpool Health Authorities for their contribution and support.

\section{References}

Alpay, L., Needham, G. and Murray, P. 2000: The potential of information technology for nurses in primary care: a review of issues and trends. Primary Health Care Research and Development 1, 5-13.

Atkin, K., Hitsch, M., Lunt, N. and Parker, G. 1994: The role and self-reported training needs of nurses employed in general practice: observations from a national census of PNs in England and Wales. Journal of Advanced Nursing 20, 46-52.

Atkin, K. and Lunt, N. 1996: Negotiating the role of the PN in general practice. Journal of Advanced Nursing 24, 498-505.

Black, S. and Hagel, D. 1996: Developing an integrated nursing team approach. Health Visitor 69, 280-3.

Bradshaw, P.L. 1999: A service in crisis? Reflection on the shortage of nurses in the British National Health Service. Journal of Nursing Management 7, 129-32.

Bull, J. 1998: Integrated nursing: A review of the literature. British Journal of Community Nursing 3, 124-9.

Cain, P., Hyde, V. and Hawkins, E. 1995: Community nursing: dimensions and dilemmas. London: Arnold.

Choppin, J. 1997: The learning organisation. Managing Service Quality 7, 269-73.

Department of Health 1997: The new NHS: modern, dependable. London: HMSO.

Department of Health 1998a: A first class service. London: HMSO.

Department of Health 1998b: Information for health - an information strategy for the modern NHS 1998-2005. Wetherby: NHS Executive.

Department of Health 1999a: Saving Lives: Our Healthier Nation. London: HMSO.

Department of Health 1999b: Making a Difference. London: HMSO.

Department of Health 2000: The NHS Plan. London: HMSO.

Emery, F. and Trist, E. 1965: The causal texture of organizational environments. Human Relations 18, 21-32.

Field, R. and West, M. 1995: Teamworking in primary health care: 2 Perspectives from practice. Journal of Interprofessional Care 9, 123-30.

Gerrish, K. 1999: Teamworking in primary care: an evaluation of the contribution of integrated nursing teams. Health and Social Care in the Community 7, 367-75.

Herberlein, T.A. and Baumgartner, R. 1978: Factors affecting response rates to mailed questionnaires: A quantitative analysis of the published literature. American Sociological Review 43, $447-62$.

Hirst, M., Atkin, K. and Lunt, N. 1995: Variations on practice nursing: implications for family health services authorities. Health and Social Care in the Community 3, 83-97.

Hyde, J. and Wright, A. 1997: Self development. Nursing Management 4, 10-11. 
Jones, D. Edwards, J. and Lester, C. 1997: The changing role of the PN. Health and Social Care in the Community 5, 77-83.

Kanuk, L. and Berensen, C. 1995: Mail surveys and response rates: A literature review. Journal of Marketing Research 12, 440-53.

Kernick, D.P. 1999: Nurses and doctors in primary care: decisions should be based on maximizing the cost effectiveness of a system of primary care and not the dictates of historical precedent. British Journal of General Practice 49, 647-9.

Iphofen, R. and Poland, F. 1998: Sociology in practice for health care professionals. London: Macmillan Press.

Laurence, J. 2000: Top Oxford heart hospital is 'riven by clashing egos'. The Independent, 16 November.

Lewis, J. 1999: The concepts of community care and primary care in the UK: the 1960s-1990s. Health and Social Care in the Community 7, 333-41.

Miller, C., Ross, N. and Freeman, M. 1999: Shared learning and clinical teamwork: new directions in education for multiprofessional practice. London: ENB.

Miller, P., Craig, N., Scott, A., Walker, A. and Hanlon, P. 1999: Measuring progress towards a primary care-led NHS. British Journal of General Practice 49, 541-5.

NHSE 1998a: Information for health care. Leeds: NHS Publications Unit.

NHSE 1998b: Working together - securing a quality workforce for the NHS. Leeds: NHS Publications Unit.

Noakes, B. and Johnson, N. 1999: Don't leave me this way. Health Service Journal 109, 20-2.

Norman, I., Peck E. and Richards, H. 1998: Interprofessional working in adult community mental health services: Setting a positive agenda. London: King's Fund.

Northway, R. and Walker, G. 1999: Promoting collaboration within community health care nursing. Journal of Community Nursing 13, 4-8.

Oughtibridge, D. 1998: Under the thumb. Nursing Management 4, 22-4.

Pearson, P. and Spencer, J. 1997: Promoting teamwork in Primary care: A research-based approach. London: Arnold.

Pong, R. 1997: Towards developing a flexible health workforce: a conference background paper. Canadian Journal of Medical Radiation Technology 28, 11-8.

Rapson, M.F. and Rice, R.B. 1999: Colleagues in caring: regional collaboratives for nursing workforce development. Educational policy implications. Journal of Nursing Education 38, 197-9.

Rashid, A., Watts, A. and Lenehan, C. 1996: Skill mix in primary care: sharing clinical workload and understanding professional roles. British Journal of General Practice 46, 639-40.

Rivett, G. 1998: From the cradle to the grave. London: Kings Fund.

Roberts, G. and Fielding, P. 1999: No vacancies. Nursing Standard 13, 22.

Ross, F. and Mackenzie, A. 1996: Nursing in primary health care: Policy into practice. London: Routledge.

Royal College of Nursing 1999: Nursing pay - facts and figures. $R C N$ briefing. London: RCN.

Smadu, M. 1996: So, what makes you want to be a nurse? Saskatchewan Registered Nurse's Association, 32-3.

Stark, S., Stronach, I., Warne, T., Skidmore, D., Cotton, A. and Montgomery, M. 2000: Teamworking in mental health: zones of comfort and challenge. ENB Research Report Series, Researching Professional Education, London: ENB.

Stark, S., Warne, T. and Street, C. 2001: Practice nursing: an evaluation of a training practice initiative. Nurse Education Today 21, 287-96.

Steel, J.E. 1999: Primary care for the 21st century. Journal of Nursing Management 7, 67-9.

Thomas, R.V.R. and Corney, R.H. 1993: Teamwork in primary care: the PN perspective. Journal of Interprofessional Care 7, 47-55.

Thomas, S. 1997: Primary care: the future. Journal of Community Nursing 11, 23-4.

UKCC 1992: The scope of professional practice. London: UKCC.

Walsh, M. 1998: Models and critical pathways in clinical nursing. London: Baillière Tindall.

Warne, T. 1998: The new NHS: The changes, challenges and opportunities. Journal of Child Health Care 2, $7-11$.

Wilcox, R. and Ruffell, T. 1997: The critical need for a focused approach to commissioning primary care development. Primary Care 7, 9-12.

Wiles, R. and Robinson, J. 1994: Teamwork in primary care: the views and experiences of nurses, midwives and HVs. Journal of Advanced Nursing 20, 324-30.

Young, L. 1997: Improved primary health care through integrated nursing. Primary Health Care 7, 10. 